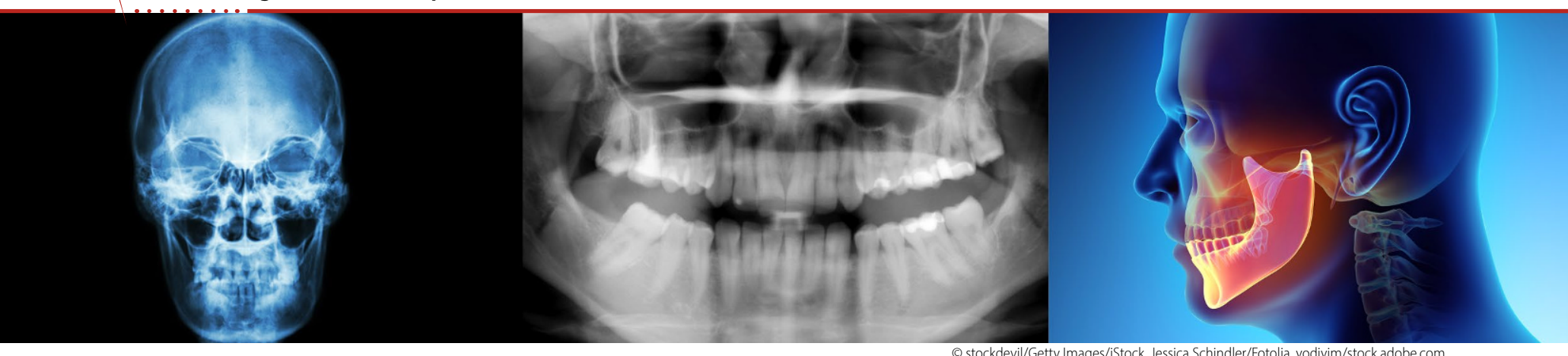

(c) stockdevil/Getty Images/iStock, Jessica Schindler/Fotolia, yodiyim/stock.adobe.com

\author{
Siddhartha Popat
}

St. Katharinen, Deutschland

\title{
Gesichts- und Kopfschmerz
}

Die Behandlung von Patienten mit Schmerzen im Kopf- und Gesichtsbereich ist in der täglichen Praxis häufig, aber leider auch immer wieder sehr schwierig.

Die Tatsache, dass es sich auch für den Humanmediziner bewährt hat, sich mit den Zähnen und dem Biss zu beschäftigen, ist in den vergangenen Jahren oft berichtet worden.

Der Doyen der Applied Kinesiology, Dr. med. Hans Garten, beispielsweise hat sich dieses Themas schon lange angenommen. Prof. Stefan Kopp, Direktor der Kieferorthopädischen Klinik der Universität Frankfurt, arbeitet sehr erfolgreich mit einer Schienentherapie, welche nach osteopathischer Vorbehandlung stetig angepasst wird.

In meiner Erfahrung hat sich folgendes Vorgehen etabliert: Sollte im Verlauf einer Behandlungserie mit Körperakupunktur trotz Anwendung verschiedener Methoden (z. B. Zang Fu, myofasziale Triggerpunkte, Segementakupunktur) keine deutliche Schmerzreduktion erreicht werden, untersuche ich den Patienten etwas anders. Die Palpation der Nackenreflexpunkte nach Adler und Langer erlaubt sehr schnell eine Verdachtsdiagnose, ob es ein Problem der Zähne geben könnte. Die Palpation der Kiefergelenke beim Öffnen und Schließen des Munds erlaubt eine Orientierung des Zustands der Kieferrelation. Dann wird dem Patienten bei Vor-

Deutsche Zeitschrift für Akupunktur

$2018 \cdot 61$ (2): 82

https://doi.org/10.1007/s42212-018-0051-x

Online publiziert: 25. April 2018

C Springer Medizin Verlag $\mathrm{GmbH}$, ein Teil von

Springer Nature 2018 liegen eines solchen Befunds die Anfertigung einer OPG-Aufnahme empfohlen und eine Wiedervorstellung verabredet.

Die ausführliche Darstellung, wie genau und wie detailliert dann der Kollege Stamer vorgeht, war ein lange gehegter Wunsch von mir. Nicolás Stamer ist mir als Dozent der DGfAN natürlich schon lange bekannt. Im Januar dieses Jahres hatte ich dann endlich die Gelegenheit, in Leipzig 2 Tage seinem Unterricht beizuwohnen, bei dem er uns die folgenden Ansätze praktisch vermittelt hat. Die Kombination von den (Nichtakupunktur-)Methoden Osteopathie, Neuraltherapie, Schienentherapie und dem Einschleifen der Zähne bis zum Erreichen des „perfekten“ Bisses sind die beste Grundlage, um mit der Akupunktur zum durchschlagenden Erfolg zu kommen!

In der Praxis habe ich das Gelernte dann natürlich sofort bei meinen Patienten eingesetzt und es ergaben sich noch schnellere und nachhaltigere Verbesserungen. $\mathrm{Da}$ nicht alle Menschen die Neuraltherapie mögen, nenne ich hier beispielhaft den Fall eines 11-jährigen Mädchens, welches nach 3 Jahren Kopfschmerzhistorie und (fach-)ärztlicher Odyssee bei mir vorstellig wurde und nach der zweiten Sitzung Akupunktur mit der Low-Level-Lasertherapie nun seit Wochen völlig schmerzfrei ist. Hierbei habe ich neben den üblichen Akupunkturpunkten mithilfe eines speziell dafür gebauten Dentalhandstücks eben auch die Störfelder der Mandeln mit dem Laser behandelt und im Anschluss mit den weichen osteopathischen Techniken nach Stamer die Kopfgelenke mobilisiert, die 1. Rippe und die HWS eingerichtet.

Mit der Vorstellung, das durch den Einsatz von Lokalanästhetika an den neuromodulativen Triggern NNH, Zähnen und Mandeln eine sofortige Besserung der Kopfgelenksfehlstellung möglich ist und dadurch periphere Reizzustände gelöscht werden, hat sich die DZA schon häufig in den letzten Jahren beschäftigt. Wichtig ist mir als Akupunkteur, dass durch die Akupunktur nun die Aktivierung der absteigenden Hemmung greift, welche den Patienten dann schmerzfrei bleiben lässt.

In diesem Kontext kommt die Akupunktur nicht zu kurz. Tom Ots zeigt anhand der Betrachtung von Di 4, dass sich die in den letzten Jahren hervorragend von Frau Kollegin Wancura-Kampik herausgearbeitete Segmentanatomie und -akupunktur erfreulich verbreitet.

Ich freue mich, dass ich als für die Related Techniques verantwortlicher Redakteur im neuen DZA-Team gleich ein solch zentrales Thema betreuen durfte, und wünsche Ihnen viel Freude und Erkenntnisgewinn beim Lesen.

Für Rückmeldungen und Kommentare sind wir in der Redaktion immer dankbar und offen!

Herzlichst,

Ihr Siddhartha Popat

Präsident der DGfAN

\section{Korrespondenzadresse}

\section{Dr. med. Siddhartha Popat}

Kolpingstraße 8, 53562 St. Katharinen,

Deutschland

sidpopat@aol.com

Interessenkonflikt. S. Popat gibt an, dass kein Interessenkonflikt besteht. 